\title{
Exploration on the Reform of Wireless Sensor Network Teaching Methods
}

\author{
Ding Li \\ Health Services Administration \\ Xi an Medical University, \\ Xi'an,710021, Shaanxi, China \\ e-mail:370486946@qq.com
}

Wang Zhongsheng

School of Computer Science and Engineering

Xi'an Technological University,

Xi'an,710021, Shaanxi, China

e-mail: 59483672@qq.com

\author{
Yan Jin \\ Department of Medical Technology \\ Xi`an Medical University, \\ Xi'an,710021, Shaanxi, China \\ e-mail: 308614756@qq.com
}

\begin{abstract}
Wireless Sensor Networks as the internet of things is one of the key technology for application, it has a wide range of applications in the future. In the traditional teaching of Wireless Sensor Networks, teachers tend to focus on the basic theory, and explain the basic methods. This teaching method ignores the importance of practice and performance, which led to a lack of students design thinking and innovation. The course through a comprehensive lab as the main line, combined with the basic theories and concepts to enable students to explore the complex lab, students not only mastered the basic knowledge, but also improve the awareness of innovation in the process of exploring complex experiments.
\end{abstract}

Keywords-Mission-driven; Practice Teaching; Education Reform

\section{INTRODUCTION}

Wireless Sensor Networks (WSN) is a wireless network system composed of a large number of lower cost sensor nodes in an ad-hoc manner. The sensor nodes deployed in the target area, the nodes through wireless communication spontaneous formation of multi-hop wireless network. The environmental information of monitoring area through the sensor to perceive, it can collect the information and processing the data, which arrived the monitoring center or end-user by the wireless network [1]. Design a complete wireless sensor networks monitoring system as part of education, the system is broken down into a number of teaching units, each unit clearly defines what needs to be done, it arouse the enthusiasm of the students. This is a mission-driven teaching method. In the process of completing the task, students not only master the basic knowledge of textbooks, but also improve the application capabilities.

\section{THE COURSE FEATURES OF 'WIRELESS SENSOR NETWORK'}

The wireless sensor network course content-rich, mainly covers the physical layer design, the network routing protocol, the technical data integration, and other aspects. This course is a multidisciplinary cross-of course; student is required to grasp the knowledge of sensor technology, wireless communications, embedded knowledge and other related knowledge. The courses require a higher degree of students that need to have a solid foundation for theoretical knowledge and comprehensive professional knowledge [2].

\section{THE REFORM OF THE TEACHING METHODS.}

According to the characteristics of the course, combined with the actual situation of student, design a number of typical based sample programs, each program are concerned 
with the individual elements of knowledge. Students master various aspects of knowledge in the design of sample programs. Before the class, the teacher explains the functions which the system needs to realize, guides students to design methods, carefully explain the basic knowledge involved in this lesson. Students discuss in groups and complete the specific requirements for this lesson. The teacher gives guidance to students and answers questions that students cannot solve. Through a concrete analysis of a sample, this article shows students through the instance of the specific knowledge that students have mastered and the effect achieved [3].

\section{Course Teaching Method Design}

The design of a monitoring system, the system architecture consists of sensor node, coordinator node and terminal computer system, its system composition is as shown in Figure 1. A large number of sensor nodes are uniformly dispersed in the measured region. When the node is energized, data information is automatically collected, and the information is transmitted to the coordinator node after the multi-hop route, the data is transmitted to the terminal system via the coordinator node [4]. In the teaching, design of sensor node, construction of network, and the design of the coordinator are the key points of teaching, analysis of each of the functions required to guide students to learn.

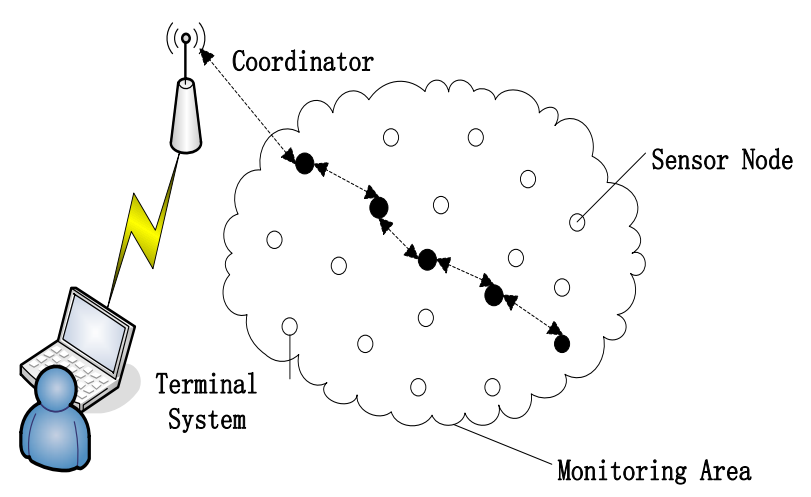

Figure 1. WSN System Composition

The sensor node has network terminal node function and routing function, the node can not only process the data information of the nodes, but also forward the data transmitted from other nodes to accomplish certain tasks. The aggregation node is mainly responsible for the data collection of other sensor nodes, and then directly uploaded to the PC monitoring terminal through standard interface.

The system needs to design the sensor node and the coordinator. The system requires students to master the sensor nodes to collect information; the node consists of four function modules: sensing module, data processing module, ZigBee network transmission module and power module, perception module consists of temperature sensor DS18B20 and humidity sensor SHT11, its structure is as shown in Figure 2. The characteristics of the sensor for sensing monitoring targets and phenomenon, and generate the corresponding analog signal, through $\mathrm{A} / \mathrm{D}$ converter is responsible for converting analog signals into digital signals, and then will be sending the digital signal processing module for processing. The processing module adopts STC89C52, which is responsible for processing the data and controlling the sensor nodes. The communication module USES ZigBee wireless single chip development kit (CC2530), it is used to send, receive data and control information. The power module USES the dry battery to power the sensor nodes. Through the study of this part, the students mastered the acquisition of sensor information and learned how to build ZigBee wireless sensor network [5].

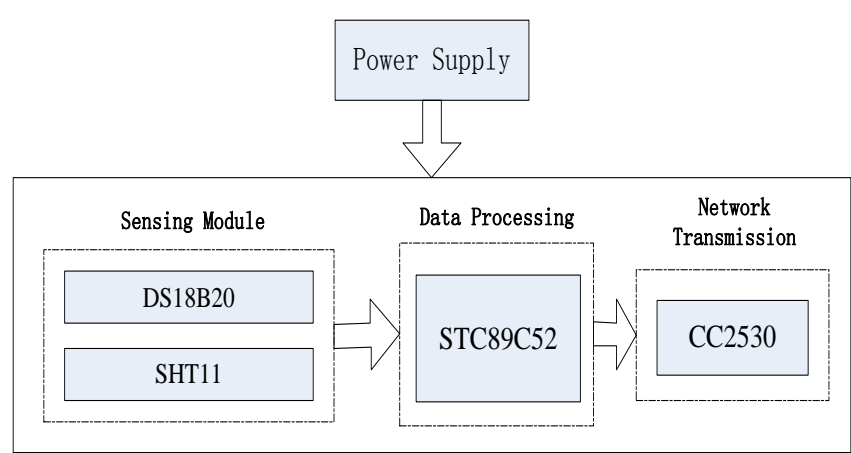

Figure 2. Data Acquisition Node Structure Diagram

The main task of the data processing module is to receive the information collected by the sensor node, and then send the information through the ZigBee module. Process 
information processing and data communication need to ensure data integrity, security and accuracy. After the device is energized, the device is initialized, the timer, input/output tube foot, communication rate, etc., then clear the buffer, wake up the sensor, and let the sensor perform the data collection work. Data is transferred to a single chip by the $\mathrm{I} 2 \mathrm{C}$ bus, then the $\mathrm{I} 2 \mathrm{C}$ bus is released and the data is transmitted to the ZigBee module via RS232 serial port. The program is designed to be infinite loop, and as long as the power is supplied, the equipment will operate. In the process of designing the data processing module, the students combined the functional requirements of the system and consolidated the knowledge content of SCM programming.

When the sensor is sent through the ZigBee module, the data will be divided into several packets according to the requirements of the ZigBee network, and each packet will be sent in order. The coordinator node receives the incoming data in order and sends the data to the upper machine for decoding to complete the communication process of the entire network. In the process of design, students have mastered the knowledge of the wireless sensor network, and have a deeper understanding of the communication protocol of the wireless sensor network.

\section{CONCLUSION}

The 'Wireless Sensor Network' course is theoretical and practical. By improving teaching method, the abstract concept is embodied in practical design. The students' knowledge of this part of the knowledge is stronger, and the interest in the curriculum has increased significantly. This teaching method not only improves the teaching effect, but also cultivates the students' ability to learn independently, which has consolidated the basic knowledge and improved the ability of application and research. In the future, we will continue to make innovations in teaching to meet the needs of cultivating innovative talents in colleges and universities.

\section{REFERENCES}

[1] WU Di, ZHU Jin-xiu. Teaching Expoloration of the Wireless Sensor Networks Course[J]JOURNAL OF EEE. 2014.08

[2] HE Dao-de, JIANG Tao. Research on Teaching Design of Wireless Sensor Networks based on Flipped Classroom [J] Computer Knowledge and Technology.2017.02

[3] WANG Chao. Discussion on teaching reform of "Zigbee protocol and programming" of Internet of things engineering $[\mathrm{J}]$ Journal of Jilin Province Economic Management Cadre College,2016.03

[4] YANG Jian-qiang, Zhang Qi-lin. Based on ZigBee's wireless sensor network course, teaching design and performance [J].Computer Education,2017.07

[5] Wu Jian, ZHANG Wei. Data collection based on wireless sensor network experiment design[J].Research and Exploration in Laboratory,2013.06 